\title{
The Impact of Inherited Retinal Diseases in the Republic of Ireland (ROI) and the United Kingdom (UK) from a Cost-of-Illness Perspective
}

This article was published in the following Dove Press journal: Clinical Ophthalmology

\author{
Orla Galvin (1D) \\ Gloria $\mathrm{Chi}^{2}$ \\ Laura Brady ${ }^{3}$ \\ Claire Hippert ${ }^{4}$ \\ Marta Del Valle Rubido ${ }^{4}$ \\ Avril Daly' \\ Michel Michaelides ${ }^{5,6}$ \\ 'Retina International, Dublin 8, D08 \\ R9CN, Ireland; ${ }^{2}$ Genentech, Roche \\ Group, South San Francisco, CA 94080- \\ 4990, USA; ${ }^{3}$ Fighting Blindness, Dublin 2, \\ D02 TW98, Ireland; ${ }^{4}$ F. Hoffmann-La \\ Roche Ltd, Basel 4070, Switzerland; \\ ${ }^{5} \mathrm{UCL}$ Institute of Ophthalmology, \\ University College London, London, UK; \\ ${ }^{6}$ Moorfields Eye Hospital NHS \\ Foundation Trust, London, UK
}

Correspondence: Orla Galvin

Email orla.galvin@retina-international.org

\begin{abstract}
To date, there has been a global lack of data regarding the prevalence of conditions falling under the Inherited Retinal Diseases (IRD) classification, the impact on the individuals and families affected, and the cost burden to economies. The absence of an international patient registry, and equitable access to genetic testing, compounds this matter. The resulting incomplete knowledge of the impact of IRDs hinders the development and commissioning of clinical services, provision of treatments, and planning and implementation of clinical trials. Thus, there is a need for stronger evidence to support value for money to regulatory bodies for treatments approved, and progressing through clinical trials. To ensure a strategic approach to future research and service provision, it is necessary to learn more about the IRD landscape. This review highlights two recent cost-of-illness reports on the socio-economic impact of 10 IRDs in the Republic of Ireland (ROI) and the United Kingdom (UK), which demonstrate the comprehensive impact of IRDs on individuals affected, their families, friends and society. Total costs attributable to IRDs in the ROI were estimated to be $£ 42.6$ million in 2019 , comprising economic (£28.8 million) and wellbeing costs ( $£ 13.8$ million). Wellbeing costs were estimated using the World Health Organization (WHO) burden of disease methodology, a non-financial approach, where pain, suffering and premature mortality are measured in terms of disability-adjusted-lifeyears (DALYs). In the UK, wellbeing costs attributable to IRDs were $£ 196.1$ million, and economic costs were $£ 327.2$ million amounting to $£ 523.3$ million total costs in 2019 . Accounting for over one-third of total costs, the wellbeing burden of persons affected by IRDs should be emphasized and factored into reimbursement processes for therapies and care pathways. This targeted review presents the most current and relevant data on IRD prevalence in the ROI and the UK, and the impacts (financial and non-financial) of IRDs in terms of diagnosis, wellbeing, employment, formal and informal care, health system costs, deadweight losses and issues surrounding payers and reimbursement. This review demonstrates IRD patients and their families have common issues including, the need for timely equitable access to genetic testing and counselling, equality in accessing employment, and a revision of the assessment process for reimbursement of therapies currently focused on the cost-of-illness to the healthcare system. This review reveals that IRD patients do not frequently engage the healthcare system and as such suggests a cost-of-illness model from a societal perspective may be a better format.
\end{abstract}

Keywords: genetic diagnosis, inherited retinal disease, cost-of-illness

\section{Introduction}

Inherited retinal diseases (IRDs) represent a diverse group of visually debilitating diseases that result in legal blindness from birth/early infancy or over time (most often in childhood or early adulthood), in which disease-causing variants in genes 
that are critical to retinal structure and/or function lead to photoreceptor cell dysfunction/death and associated vision loss. Individual IRDs are classified as rare diseases. ${ }^{1}$ In the European Union, a disease is classified as rare if it affects less than 1 in 2000 people. $^{1-3}$ In the USA, a disease is classified as rare if it affects less than 200,000 people. ${ }^{2,3}$ Due to the small numbers affected, funding for research and innovation for these conditions historically has been limited. Lack of data hinders diagnosis and clinical care for IRD patients, thus motivating the need to better describe the burden and impact of IRDs to allow for the allocation of funding and programs to improve the development of new therapies and services for patients with IRD.

This has led to a relative lack of knowledge and understanding of these diseases, which often results in a delay in diagnosis. For rare disease cases, it has been reported that diagnosis took an average of 7.6 years in the USA, and 5.6 years in the UK, during which time patients typically visited eight physicians, four primary and four specialists, and received two to three misdiagnoses. ${ }^{2}$ While significant advances have been made to identify over 300 genes responsible for IRDs, ${ }^{4}$ knowledge gaps remain to identify the remaining causative genes, improve genetic testing methodologies, and moreover access to them. ${ }^{5}$ Indeed, insufficient data on prevalence have contributed to the insufficient funding and resources available to conduct genetic testing for IRDs, and to provide genetic counselling for IRD patients and families. This has resulted in many patients waiting years to obtain genetic test results.

The development of treatments and cures has also been limited to date, with only one recently approved therapy for a childhood-onset IRD (Luxturna ${ }^{6}$ ).

These conditions have imposed a considerable emotional toll on patients and their care-givers. ${ }^{2,7-9}$ Other challenges include a lack of information regarding the natural history of the majority of IRDs, resources, and the financial cost of informal and formal care. ${ }^{2}$ Accessing appropriate medical expertise, which is compounded by a lack of specialist training programmes for medical professionals on IRDs, ${ }^{2}$ coupled with very low genetic testing rates, ${ }^{7,8}$ underlies the delay in obtaining a correct diagnosis and appropriate counselling/support.

A targeted literature review was carried out to allow the most current and relevant data pertaining to the impact and burden of IRDs in the UK and ROI to be included in this review. The objectives of this review are to demonstrate and improve awareness of how the incorporation of a patient- centered approach is essential to ensure that the priorities of researchers, clinicians, funders and policymakers are aligned to the needs and priorities of those who experience retinal degeneration: the patient, and those who care for them. People increasingly want to be informed, empowered, and engaged with their medical management. ${ }^{10}$ A patientcentered approach means that patients are not just passive subjects in clinical research anymore, but active participants, and engaged stakeholders. ${ }^{11}$

Important features of this review include the outline of key findings of the recent landmark cost-of-illness reports for IRDs in the UK and ROI, and to discuss the barriers and burdens the lack of data and awareness of IRDs have to the IRD community and to society.

This review presents data, which can be used to define the economic burden of a disease, justify investment in preventive or treatment interventions, inform funding allocation and prioritisation, provide a basis for policy and planning, and provide inputs for economic analyses. However, there is still a need for further more comprehensive data.

This review serves to fill some of the aforementioned knowledge gaps and presents data from cost-of-illness reports for the ROI and UK which employed a patientcentred approach in the design of surveys for real-world evidence data generation from the patients and parents involved. Patient involvement is crucial to inform decisions in the IRD space.

\section{Prevalence}

The overall prevalence of the 10 IRDs listed in Table 1 is estimated to be $0.0311 \%$ in ROI and UK for the year 2019;

Table I Estimated Prevalent Cases (Number of People) with IRDs in ROI and UK

\begin{tabular}{|l|l|l|l|l|}
\hline Region & \multicolumn{2}{|l|}{ Republic of Ireland } & \multicolumn{2}{l|}{ United Kingdom } \\
\hline IRD & Base & Upper & Base & Upper \\
& Cases & Band & Cases & Band \\
\hline Retinitis Pigmentosa & 755 & 1274 & 10,324 & 17,422 \\
Usher Syndrome & 189 & 320 & 2590 & 4371 \\
Stargardt Disease & 154 & 260 & 2109 & 3559 \\
LCA/EOSRD & 116 & 196 & 1587 & 2679 \\
Best Disease & 73 & 124 & 1003 & 1693 \\
Cone Dystrophy & 64 & 108 & 876 & 1478 \\
Cone Rod Dystrophy & 59 & 100 & 812 & 1370 \\
Achromatopsia & 54 & 90 & 733 & 1237 \\
X-linked Retinoschisis & 35 & 60 & 483 & 814 \\
Choroideremia & 22 & 37 & 298 & 504 \\
\hline Total & 1,521 & 2,569 & 20,815 & 35,126 \\
\hline
\end{tabular}


methods and prevalence breakdown by region (ROI and UK), age and sex are published in the reports. ${ }^{7,8}$ In many cases, the inputs underlying a cost-of-illness analysis are uncertain and changes in these inputs may have a significant impact upon the total estimate of the costs of IRDs in the ROI and UK in 2019. Given the limited published data on the prevalence of IRDs in the ROI and UK, a sensitivity analysis using an upper bound sensitivity estimate based on the highest published prevalence estimate available was undertaken. Using this approach, an upper bound prevalence rate of $0.0525 \%$ was estimated for the ROI and UK - $68.8 \%$ higher than the base prevalence estimate of $0.0311 \%$ reported in this analysis. ${ }^{7,8}$ While this source reports that its underlying research is based on a systematic review of PubMed, grey literature, and expert consultation, primary data informing these estimates are difficult to ascertain and verify, and therefore does not inform the base estimates or values presented in the review.

Table 1 indicates the base case and upper band prevalence estimates across the 10 IRDs in the ROI and the UK for 2019. The total base to upper band estimates range for the 10 IRDs in the ROI is 1521-2569 prevalent cases in 2019, and in the UK, this range is 20,815-35,126 prevalent cases.

\section{Diagnosis and Genetic Testing}

Accurate diagnosis requires molecular genetic testing. The clinical diagnosis is likely to be made by a comprehensive ophthalmologist, pediatric ophthalmologist, or retinal specialist. $^{12}$ A number of questions will arise at clinical diagnosis. Who should have genetic testing? What difference will the results of molecular genetic testing make to clinical management? Who will pay for testing? Molecular diagnosis will not only confirm the clinical diagnosis but also identify the correct inheritance pattern and guide accurate genetic counselling for the patient and their family (including potentially preimplantation genetic diagnosis). ${ }^{12}$ It will also help to inform prognosis, which is particularly important in younger subjects, especially in the case of children who are at risk of systemic problems. ${ }^{12}$ An accurate molecular diagnosis can inform dietary decisions such as vitamins and minerals which may be of benefit and which to avoid. ${ }^{13,14}$ For example, high intake of vitamin $\mathrm{A}$ is a suspected risk factor for disease progression for those affected by Stargardt's disease, ${ }^{14}$ indeed individuals with Stargardt's disease with low dietary vitamin A intake showed significantly better visual acuity with respect to those introducing higher intake of vitamin A. ${ }^{15}$ In contrast, supplementation of vitamin A has been shown to have protective effects delaying progression of Retinitis Pigmentosa. ${ }^{13,15}$ In addition, obtaining a molecular diagnosis informs patients and clinicians of their potential suitability for participation in clinical trials.

Multiple clinical trials to treat IRDs are ongoing, with the first gene therapy for IRD, Luxturna, having been approved for use. ${ }^{6}$ A 4-year follow-up report indicates stable, persistent improvement in tests of functional vision and visual function such as navigational ability and light sensitivity in subjects with RPE65 mutation-associated IRD. ${ }^{16}$ Typically, such trials and treatments require that a causative variant has been identified in order to participate. Thus, genetic testing empowers patients with genetic diagnoses to have potential future access to clinical trials or approved treatments where appropriate. Given the rapid development of the field of ocular therapeutics, genetically characterising those affected by an IRD has become a diagnostic imperative. ${ }^{17}$

Recruitment to clinical trials depends on there being sufficient numbers of patients with these rare IRDs who know their specific disease-causing sequence variant. Subsequently, as these life-changing therapies continue to reach the clinic, it will be critical to identify patients who may benefit, ${ }^{12}$ and reduce the economic and wellbeing burden of IRDs to families and society as a whole. Genetic testing will allow for the development of patient registries, thus enabling the description of natural histories of the conditions, determining clinical and/or costeffectiveness, assessing safety/harm, and measuring or improving quality of care. ${ }^{18}$

However, it is important to be aware that the information revealed from a genetic test may not be immediately beneficial to an individual or family. The result may create anxiety for some family members, or may not reveal the defective gene, which can be frustrating. It is therefore firmly recommended that a genetic test for an IRD is only carried out in conjunction with a genetic counsellor or retinal specialist who is knowledgeable about the genetic testing process and the potential impacts of the results.

Reports analysing the cost-of-illness of 10 IRDs in the ROI and the UK were carried out for the year 2019. ${ }^{7,8}$ The 10 IRDs included in the study were Retinitis Pigmentosa (RP), Leber Congenital Amaurosis (LCA) and Early Onset Severe Retinal Dystrophy (EOSRD), Cone Dystrophy, Choroideremia, Usher Syndrome, Best Disease, Cone-Rod Dystrophy, Stargardt Disease, X-Linked Retinoschisis 
(XLRS), and Achromatopsia. All of these conditions can be tested for by molecular genetic techniques. A description of each condition is detailed in the Cost-of-illness (COI) reports in Section 2. ${ }^{7,8}$

A survey of persons living with an IRD in the ROI and UK, along with parents/guardians of children under the age of 18 , was carried out in the ROI and the UK. ${ }^{7,8}$ There were 129 participants which reflected 129 unique patients, representing $60 \%$ uptake of the survey. Of these 76 were based in the ROI and 53 were based in the UK. Of the 76 ROI participants, 52 were aged 18 or older and were themselves living with an IRD. The remaining 24 were the parent or guardian of a child under the age of 18 living with an IRD. Of the 53 participants in the UK, 44 were aged 18 or older and were themselves living with an IRD. The remaining 9 were the parent or guardian of a child under the age of 18 living with an IRD. ${ }^{7,8}$

For the purpose of accurately diagnosing their IRD, $59.2 \%$ of survey participants in the ROI, and $56.6 \%$ of participants in the UK, reported they had undergone a genetic test; however, this does not indicate a received genetic test result. ${ }^{7,8}$ Survey participants in the ROI indicate $66 \%$ of family members underwent genetic testing for the IRD, while the corresponding figure in the UK was $24.5 \%{ }^{7,8}$ For the purpose of the COI surveys, the rationale as to why patients had not had genetic testing was not investigated.

A summary of the survey responses in relation to genetic testing and genetic counselling in the $\mathrm{ROI}^{7}$ and the $\mathrm{UK}^{8}$ is outlined in Table 2.

While the uptake of genetic testing is low and the provision of genetic counselling limited, some insight can be gained from recent research into the perceptions of parents regarding their children's diagnostic testing for IRDs in China. Almost half of parents supported genetic testing to help in making informed reproductive health decisions, to

Table 2 Survey Responses in Relation to Genetic Testing and Genetic Counselling

\begin{tabular}{|l|l|l|}
\hline $\begin{array}{l}\text { Survey Responses in Relation to Genetic } \\
\text { Testing and Genetic Counselling }\end{array}$ & ROI & UK \\
\hline Received a genetic test in their lifetime* & $59.2 \%$ & $56.6 \%$ \\
Never received genetic counselling & $69.7 \%$ & $50.9 \%$ \\
Received genetic test in last I2 months* & $22.2 \%$ & $26.7 \%$ \\
Received genetic counselling in last I2 months & $10.9 \%$ & $8.3 \%$ \\
Family member received a genetic test* & $66.0 \%$ & $24.5 \%$ \\
Family member received genetic counselling & $33.0 \%$ & $14.8 \%$ \\
\hline
\end{tabular}

Note: *Undergoing a genetic test does not indicate receipt of a genetic test result. prepare for novel potential treatment, to identify the underlying causes of IRDs, and to satisfy curiosity about the heredity of IRDs (given in approximately $50 \%$ of cases, there is no definite family history of IRD - the genetic basis is often (wrongly but understandably) questioned by patients and families). ${ }^{19}$ However, $19.6 \%$ were opposed to testing for four main reasons, namely (i) lack of therapeutic benefit, (ii) difficulty in affording the testing cost, (iii) doubt in the accuracy of clinical diagnosis, and (iv) concerns about the limitations of genetic testing. ${ }^{19}$ While the remaining $32.6 \%$ expressed that they would be willing to receive genetic testing if (i) the doctors highly recommended it, (ii) the results would be helpful to the treatment, or (iii) the testing was affordable. Almost half of the parents expressed concerns that the genetic findings might lead to potential psychological stress in the family. ${ }^{19}$ Inheritance of a genetic disorder may invoke many technical, ethical and highly personal questions, including feelings of guilt and helplessness. Therefore, genetic counselling is essential, including providing accurate information and guidance, and offering further support services within a clinical setting. These professionals assist patients in addressing the scientific and emotional issues that arise in such situations and help them make informed decisions based on their own values and individual circumstances. $^{20}$

As more therapies progress through clinical trials there may be an associated increase in the uptake of genetic testing for IRDs, the authors acknowledge this would be associated with an increase in genetic testing costs. However, over time this may in part be counteracted by the decreasing costs of whole genome and whole exome sequencing. ${ }^{17}$ An increase in genetic testing should coincide with an increase in genetic counselling incurring additional costs, it is estimated that the unit cost for one visit to a genetic counsellor is $£ 185$ in the ROI and $£ 172$ in the UK. ${ }^{7,8}$

\section{Attributing Costs to IRDs}

The cost-of-illness reports followed a cost-of-illness methodology applying a prevalence approach which involves estimating the number of persons living with an IRD in a base period (2019) and the costs attributable to IRDs in that period. A summary of the overall costs by condition based on prevalence estimates according to both reports is outlined in Table 3.

The ROI and UK cost-of-illness reports on IRDs estimated first-round societal impacts. No second round or longer term dynamic impacts were modelled (ie changes 
Table 3 Cost Associated with Individual IRDs Based on Base Case Prevalence Estimates

\begin{tabular}{|l|l|l|}
\hline Region & ROI & UK \\
\hline IRD & € Million & € Million \\
\hline RP & 21.4 & 262.3 \\
Usher syndrome & 5.3 & 64.9 \\
Stargardt disease & 4.2 & 52.0 \\
LCA/EOSRD & 3.1 & 38.5 \\
Best disease & 2.0 & 24.8 \\
Cone dystrophy & 1.8 & 22.1 \\
Cone-rod dystrophy & 1.6 & 20.5 \\
Achromatopsia & 1.5 & 18.1 \\
XLRS & 1.0 & 12.6 \\
Choroideremia & 0.6 & 7.5 \\
\hline Total & 42.6 & 523.3 \\
\hline
\end{tabular}

in wages or labour market outcomes associated with the economic burden of IRDs). The five main cost categories are outlined below and relate to:

(i) Wellbeing costs. Loss of wellbeing was measured by years of healthy life lost to IRDs. This is a nonfinancial approach, where pain, suffering and premature mortality are measured in terms of disability-adjusted life years (DALYs). Disability weights were applied to the prevalence of IRDs in the ROI and the UK. DALYs were converted to euros and pounds in the COI reports using an estimate of the value of a statistical life year (VSLY). For full methodology see Section 9.1 of COI reports. ${ }^{7,8}$

(ii) Individual productivity losses were calculated as reduced workforce participation, absenteeism and presenteeism (reduced productivity while at work) of those living with an IRD, relative to the general population. For full methodology see Section 7 of the COI reports. ${ }^{7,8}$

(iii) Formal and informal care was estimated on the number of individuals with an IRD that receive formal and/or informal care, the number of hours of care, and the cost per hour. For full methodology see Section 8.5 of COI reports. ${ }^{7,8}$

(iv) Costs to the health system include primary and secondary health care, pharmaceuticals, vitamins and supplements, diagnostic tests and medical research.

(v) Deadweight losses represent society-wide efficiency losses arising from loss of taxation revenue due to reduced workforce participation and higher government expenditures.
A targeted review of the medical journal databases PubMed and Cochrane library covering the period January 1, 2010 to December 31, 2018, and publicly available databases were conducted to identify the most relevant inputs for the cost-of-illness reports. ${ }^{7,8}$ Published data were supported by primary data collection through a survey of the persons over 18 living with one of the 10 IRDs listed, or the parents/guardians of children under 18 who are living with an IRD listed in these reports.

A summary of the overall costs by category according to both reports is outlined in Table 4. Of interest, the highest cost type associated with IRDs in both regions was due to wellbeing costs, followed second in both regions by productivity costs; with the lowest cost in both regions being health system costs, indicative of the low level of interaction of IRD patients with the health systems.

\section{Wellbeing}

Wellbeing is a keyword in the WHO's definition of health: "a state of complete physical, mental and social wellbeing and not merely the absence of disease or infirmity".

Research has proven what the patient organisations (and clinicians) have known anecdotally. Profound vision loss in adolescents, young and middle-aged adults is associated with significant negative psychological and psychosocial effects. $^{21}$ Interestingly, psychological wellbeing was assessed as mood, quality of an individual's interpersonal interactions, and career goals. ${ }^{21}$ The impact of visual impairment on career and occupational goals should be of high relevance to ophthalmologists and researchers, although few studies have examined these effects. Impaired vision-related quality of life has been shown to be associated with the inability to work and low socioeconomic status. ${ }^{22}$ The impact of IRDs on participation in the workforce in the

Table 4 Breakdown of Cost Type Based on Base Case Prevalence Estimates

\begin{tabular}{|l|l|l|l|l|}
\hline Region & \multicolumn{2}{l|}{ ROI } & \multicolumn{2}{l|}{ UK } \\
\hline Cost Type & \% Total & f Million & \% Total & f Million \\
\hline WellbeingCosts & 32.3 & 13.8 & 37.5 & 196.1 \\
Productivity Costs & 18.9 & 8.1 & 21.8 & 114.1 \\
Deadweight losses & 16.6 & 7.1 & 5.5 & 28.7 \\
Informal Carer Costs & 13.6 & 5.8 & 16.1 & 84.5 \\
Other Costs & 14.2 & 6.0 & 14.3 & 74.9 \\
Health System Costs & 4.5 & 1.9 & 4.8 & 25.0 \\
\hline Total & 100 & 42.6 & 100 & 523.3 \\
\hline
\end{tabular}


ROI and the UK is described in the productivity section below.

According to cost-of-illness reports of IRDs in the ROI and the UK, wellbeing costs comprised the largest share of total costs (Table 4), this cost is summarised in Table 5. In 2019, persons living with an IRD in the ROI were estimated to experience a total of 234 (DALYs), and in the UK 3192 DALYs.

In a survey of adults living with an IRD (Table 6), and the parents/guardians of children under 18 living with an IRD (Table 7), participants in both the ROI and the UK indicated high levels of negative mental wellbeing impacts. $^{7,8}$

Table 5 Cost of Wellbeing Associated with IRDs in the ROI and the UK

\begin{tabular}{|l|l|l|}
\hline Region & ROI & UK \\
\hline Per Person Cost & $£ 9,023$ & $£ 9,421$ \\
Total Cost & $£ \mid 3.8$ Million & $£ \mid 96.1$ Million \\
\hline
\end{tabular}

Table 6 IRD Impact on Mental Wellbeing of Persons Living with an IRD

\begin{tabular}{|c|c|c|}
\hline Region & ROI & UK \\
\hline $\begin{array}{l}\text { Wellbeing state occurring } \\
\text { as a direct result of IRD }\end{array}$ & $\begin{array}{l}\text { Percentage } \\
\text { (\%) of } \\
\text { respondents }\end{array}$ & $\begin{array}{l}\text { Percentage } \\
\text { (\%) of } \\
\text { respondents }\end{array}$ \\
\hline Anxiety & 85.5 & 86.8 \\
\hline Depression & 63.2 & 67.9 \\
\hline Social Isolation & 44.7 & 56.6 \\
\hline Financial Stress & 43.4 & 45.3 \\
\hline $\begin{array}{l}\text { Other (fear, stress, fatigue, } \\
\text { hopelessness, loneliness, panic } \\
\text { attacks, suicidal thoughts and } \\
\text { the development of tics) }\end{array}$ & 7.9 & 13.2 \\
\hline
\end{tabular}

Table 7 IRD Impact on Mental Wellbeing of Parents/Guardian of Children Under 18 Living with an IRD

\begin{tabular}{|l|l|l|}
\hline Region & ROI & UK \\
\hline $\begin{array}{l}\text { Wellbeing State Occurring } \\
\text { as a Direct Result of IRD }\end{array}$ & $\begin{array}{l}\text { Percentage } \\
\text { (\%) of } \\
\text { Respondents }\end{array}$ & $\begin{array}{l}\text { Percentage } \\
\text { (\%) of } \\
\text { Respondents }\end{array}$ \\
\hline Anxiety & 75 & 77.8 \\
$\begin{array}{l}\text { Depression } \\
\begin{array}{l}\text { Other (stress, loneliness and } \\
\text { concerns for the safety of their } \\
\text { child) }\end{array}\end{array}$ & 50 & 77.8 \\
\hline
\end{tabular}

The percentage of negative mental wellbeing states on persons living with an IRD in the ROI and the UK follow similar trends; Anxiety (85.5\%, 86.8\%); Depression $(63.2 \%, 67.9 \%)$, Social Isolation $(44.7 \%, 56.6 \%)$, Financial Stress $(43.4 \%, 45.3 \%)$ and Other $(7.9 \%$, $13.2 \%$ ); where other reflects on fear, stress, fatigue, hopelessness, loneliness, panic attacks, suicidal thoughts and the development of tics (Table 6). ${ }^{7,8}$

For parents and guardians of children under 18 living with an IRD in both the ROI and the UK negative mental wellbeing states are also high. In the ROI and UK, respectively, the percentage of respondents reporting Anxiety were $75 \%$ and $78.8 \%$, Depression $50 \%$ and $78.8 \%$, and Other $8.3 \%$ and $22.2 \%$; where other reflected stress, loneliness and concerns for the safety of their child (Table 7). ${ }^{7,8}$

In addition, the majority of persons living with an IRD (73.2\%) and the parents of children (under 18) living with an IRD (75\%) were frustrated by the lack of awareness and support for IRDs. ${ }^{7,8}$

\section{Productivity}

Persons living with an IRD may experience a reduced capacity to effectively participate in the workforce relative to the general population due to disadvantages in jobseeking or self-selection out of the labour force. Additionally, persons living with an IRD may experience presenteeism. Presenteeism is the average number of hours per day that an employee loses to reduced performance or impaired function as a result of their condition. This is measurable by a reduction in the quality and efficiency of work produced. This can lead to significant productivity losses through lost wages and lost productive time associated with IRDs. ${ }^{7,8}$ Losses associated with reduced working hours are assigned to both productivity losses, as described in this section, and deadweight losses as described below. In relation to IRDs, productivity losses include reduced participation in the workforce and presenteeism, but not absenteeism. Survey responses in both the UK and the ROI indicated there were no cases of absenteeism as a result of an IRD. Therefore, there was no productivity cost due to absenteeism assigned to employers, government, or individuals. ${ }^{7,8}$

There are differences in the employment status between respondents to the IRD surveys in the $\mathrm{ROI}^{7}$ and the $\mathrm{UK}^{8}$ that are outlined in Table 8 . As such, survey participants were employed at a rate of $45.5 \%$ compared to the general population employment rate of $76.1 \%{ }^{8}$ 
Table 8 Working or Student Status of Survey Respondents in the ROI and the UK

\begin{tabular}{|l|l|l|}
\hline Working or Student Status & ROI & UK \\
\hline Full-Time & $30.5 \%$ & $4.5 \%$ \\
Part-Time & $9.5 \%$ & $29.5 \%$ \\
Casual Work & $5.8 \%$ & $2.3 \%$ \\
Underemployed & $1.9 \%$ & $15.9 \%$ \\
Not Employed and not looking for work & $19.2 \%$ & - \\
Retired & $23.1 \%$ & $38.6 \%$ \\
Students & $9.6 \%$ & $9.1 \%$ \\
\hline
\end{tabular}

In 2019, the individual productivity costs of IRDs in the ROI and the UK were estimated to be $£ 8.1$ and $£ 114.1$ million. $^{7,8}$ In the ROI and the UK, respectively, 74\% ( $£ 5.9$ million), and 78\% ( $£ 89$ million) of this was due to forgone income as a result of reduced workforce participation. ${ }^{7,8}$ In the ROI and the UK, persons with an IRD were $55.7 \%$ and $40.2 \%$, respectively, less likely to be in paid employment than the general population.

Relative to absenteeism, which was reported by participants in both regions as zero, presenteeism may occur more frequently and have a larger effect. ${ }^{23}$ Indeed, this was found to be the case in both the ROI and the UK, with an estimated $£ 2.1$ million individual productivity loss in the ROI, and $£ 25.1$ million in the UK due to presenteeism. ${ }^{7,8}$ In both regions, productivity costs were highest for people aged 45-49 years, reflecting the high earnings and employment rates in that age group, and in both regions, IRDs resulted in a $9.6 \%$ reduction in productivity while at work. ${ }^{7,8}$

Persons living with an IRD may require aids and modifications for daily living, employment and education settings. In the ROI and the UK, the cost of aids and modifications for daily living including work and/or education was $£ 1600$ and $£ 2148$ per person; with a total cost $£ 2.4$ million in the ROI and $£ 44.7$ million in the UK for 2019. ${ }^{7,8}$

In the ROI persons living with an IRD and in employment indicated, $36 \%$ use of magnifiers, $20 \%$ use of modifications to phones, $12 \%$ use of spoken word processor or screen reader and $24 \%$ indicated they did not use any additional items to support their work because it was not available to them. ${ }^{7}$ By comparison in the UK, persons living with an IRD in employment indicated $38.9 \%$ use magnifiers, $22.2 \%$ use modifications to phones and laptops, $16.7 \%$ use spoken word processor or screen reader and $5.6 \%$ reported not using additional items to support their work because it was not available to them.

\section{Formal and Informal Care}

IRDs can result in impairment and disability that not only affects those persons living with the condition but can also affect their family and friends. Individuals with severe visual impairment resulting from an IRD can have limited ability to engage in day-to-day and self-care activities, requiring others to do these activities in their place via formal or informal care.

Based on the survey responses reported by the COI reports in the UK and ROI, ${ }^{7,8}$ calculations carried out by the authors indicate in the ROI $78 \%$ of those providing informal care were female, $57 \%$ were the spouse of the individual living with an IRD, 36\% were another family member, and $7 \%$ of informal carers were a friend/other.

By comparison in the UK of those providing informal care $54.8 \%$ were female, $55 \%$ were the spouse of the individual living with an IRD, $40 \%$ were another family member and, $5 \%$ were a friend/other. ${ }^{7,8}$

To calculate formal care costs, the average number of hours were multiplied by the hourly cost of formal care in the ROI and the UK. ${ }^{24,25}$ The informal care costs were calculated by multiplying the number of informal care hours by the national average weekly earnings. ${ }^{7,8}$

Formal care can include help from a private nurse or assistance with activities such as childcare, housekeeping and shopping - these costs are not subsidised by private health insurance or the government, but instead are out-ofpocket expenses borne by the individual or their family. In the ROI $7.9 \%$ and in the UK $3.8 \%$ of survey respondents indicated they received formal care. ${ }^{7,8}$ The average weekly hours of formal care across both regions was $14.1 \mathrm{hrs}$, at a cost of $£ 22$ per hour in the UK, and $£ 17.61$ in the ROI. Overall, formal care costs were estimated to be $£ 1.5$ million in the ROI and $£ 13.1$ million in the UK.

A range of informal care activities may be provided to persons living with an IRD. Informal care activities depend on the level of visual impairment and can include: collecting relevant prescriptions and organising and timing the administration of medication; assistance in daily domestic activities such as cooking and laundry; ad-hoc tasks, such as shopping, transport and cleaning activities; monitoring of the patient's physical and mental wellbeing. The average weekly informal care given is $12.9 \mathrm{hrs}$ associated with a cost of $£ 13.10$ per hour in the UK. In the ROI the average weekly informal care given is $16.3 \mathrm{hrs}$ associated with a cost of $£ 15.05$ per hour. Of those surveyed in the ROI $55.3 \%$, and in the UK $20.8 \%$, indicated they 
received informal care. Informal carer cost in the ROI and the UK for 2019 is $£ 5.8$ million, and $£ 84.5$ million, respectively. $^{7,8}$

Persons living with an IRD who are unable to live at home may require residential services paid for by themselves or provided by voluntary organisations or the HSE in the ROI or the NHS in the UK. The total expenditure for long-term residential care due to IRDs in the ROI was $£ 1.1$ million in 2019 . $^{7}$ Of this, $£ 0.6$ million was paid for by individuals, and $£ 0.5$ million of this paid for by the government. In the UK, the total expenditure for long-term residential care in 2019 was $£ 4.6$ million. $^{8}$ Of this, $£ 2.6$ million was paid for individuals, and $£ 2.0$ million paid for by the government.

\section{Health System Costs}

Compared to the costs associated with wellbeing, the Health System Costs were relatively very low. The health system costs were based on the utilisation of services and frequency data obtained through survey. ${ }^{7,8}$ Where possible, national service utilisation, price references, and costs of pharmaceuticals were based published material. The full detail of the health system costing approach is included in the IRD cost-of -illness reports for the ROI and the UK. ${ }^{7,8}$ A summary of the health system costing approach includes primary and secondary care, diagnostic tests, pharmaceuticals, vitamins and supplements, and medical research (medical research costs were not included in the ROI report due to a lack of data).

The total health system costs for the ROI were $£ 1.9 \mathrm{M}$, and for the UK $£ 25 \mathrm{M}$. The greatest cost to the health system in both regions was secondary health care costing $£ 0.7 \mathrm{M}$ to the ROI and £9M to the UK. The lowest health system cost was vitamins and supplements, $£ 0.1 \mathrm{M}$ to the ROI and $£ 0.7 \mathrm{M}$ to the UK. Table 9 indicates the breakdown of Health System costs to both regions.

Payment for health system costs in the ROI and the UK is spread across government, out-of-pocket patient

Table 9 Health System Costs by Type in ROI and UK

\begin{tabular}{|c|c|c|}
\hline Health System Type & ROI (Million) & UK (Million) \\
\hline Primary Health Care & $£ 0.6$ & $£ 3.4$ \\
\hline Secondary Health Care & $£ 0.7$ & $£ 9.0$ \\
\hline Diagnostic Tests & $€ 0.3$ & $£ 4.2$ \\
\hline Pharmaceuticals & $£ 0.2$ & $£ 4.6$ \\
\hline Vitamins and Supplements & $€ 0.1$ & $£ 0.7$ \\
\hline Medical Research & - & $\notin 3.1$ \\
\hline Total & $\notin 1.9$ & $£ 25.0$ \\
\hline
\end{tabular}

payments and funding from other parties such as private health insurers.

\section{Deadweight Losses}

The deadweight loss due to lost taxation revenue (given an assumption of no change in spending) or additional expenditure on government programs (eg health or welfare) can be estimated by applying the marginal burden of taxation to the total of lost taxation and government expenditures. Lower employment participation and lower output (eg due to absenteeism or presenteeism) reduce the possible taxation revenue government can collect. Deadweight loss costs described below are independent of any other cost described in this review and do not form part of costs associated with any other cost type.

Deadweight losses summed to $£ 7.1$ million and $£ 28.7$ million, covering lost individual taxes, lost company taxes, welfare payments, due to reduced participation in the workforce and productive working hours; government programs and lost carer taxes, ${ }^{7,8}$ deadweight losses due to IRDs summed to $£ 4629$ per person, reflecting a total of $£ 7.1$ million in the ROI in 2019; resulting in a total reduction in taxation revenue was estimation of $£ 3.5$ million in 2019. ${ }^{7}$ Deadweight losses due to IRDs summed $£ 1379$ per person reflecting a total of $£ 28.7$ million in the UK in 2019; resulting in a total reduction in taxation revenue was the estimation of $£ 45.4$ million in 2019 . $^{8}$

\section{Who are the Payers?}

As it currently stands, persons living with an IRD and parents of children living with an IRD incur significant economic costs. In addition to the costs incurred by these persons, their families, friends, government, employers and society/other all incur significant economic costs due to IRDs.

The methodology of the COI reports for the ROI and the UK takes a societal perspective, which considers all costs and effects that flow from the IRDs, regardless of who experiences these. In terms of attributing costs to payers (as shown in Figure 1), society/other represents all the remaining costs that are not directly attributable to the government (eg welfare payments), families (eg carer costs) or individuals (eg medical expenses). This could include health insurers and society more broadly in terms of productivity and efficiency losses for the economy as a whole.

The method for attributing costs between society/other, government, family members and the individual were based on the OECD (2017) who published an estimated share of health system costs borne by the government, 
A

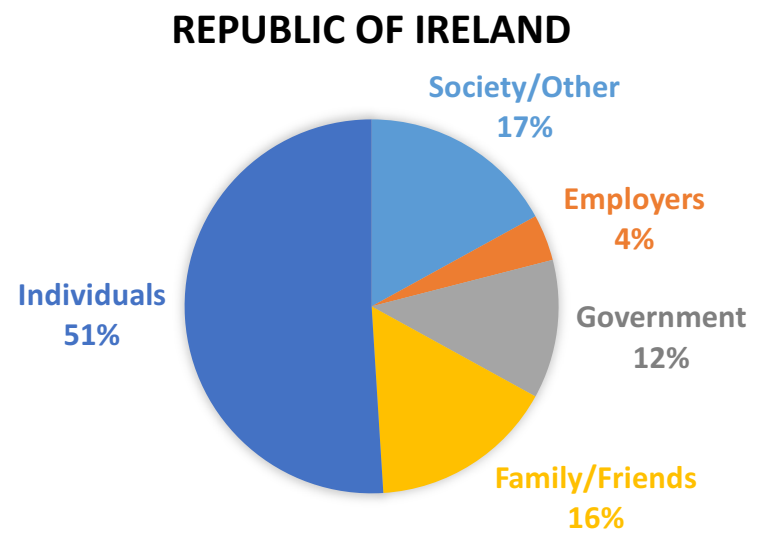

Figure I (A) Payers in the Republic of Ireland. (B) Payers in the United Kingdom.

compulsory health insurance, out-of-pocket, voluntary health insurance and other. ${ }^{26}$

Figure 1 illustrates the percentage of cost by payer in the ROI (Figure 1A) on the left pie chart and in the UK on the right pie chart (Figure 1B). In the ROI individuals living with IRDs incur the largest share of total costs at $51 \%$, followed by society/other (17\%), family/friends $(16 \%)$, government $(12 \%)$ and employers $(4 \%)$. In the $\mathrm{UK}$, individuals bear the largest share of total costs at $36 \%$, followed by government (28\%), family and friends (21\%), society/others (9\%), and employers $(6 \%)$.

\section{Discussion}

This review highlights that IRDs impose significant economic and wellbeing costs on the ROI and UK population in 2019 - which may well yet be an under-estimation. As it currently stands, persons living with an IRD incur significant economic costs and reductions in their quality of life. Using base prevalence rates, total costs attributable to IRDs in the ROI were estimated to be $£ 42.6$ million in 2019 , comprising both economic (£28.8 million) and wellbeing costs (£13.8 million). Total costs attributable to IRDs in the UK were estimated to be $£ 523.3$ million in 2019 , comprising both economic costs (£327.2 million) and wellbeing costs (£196.1 million). Thereby a total cost of $£ 42.6$ million for ROI and $£ 523.3$ million for the UK. The discussion below summarises the key messages relating to the three main issues common to IRD patient relating to accurate timely diagnosis, access to employment and the need for a revision to current methods of reimbursement assessment.

There is a need for specialised IRD testing. Due to the variety and rarity of IRDs, diagnosis can be elusive. It is often important to define the phenotype to establish the

\section{B UNITED KINGDOM}

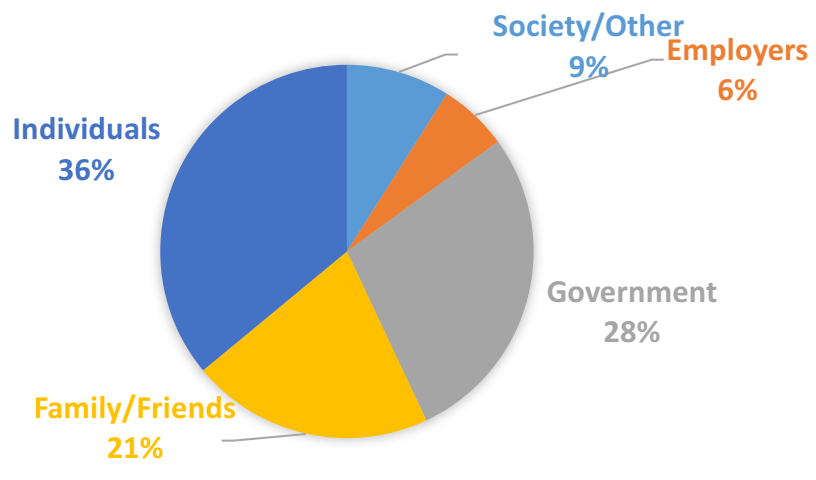

type of genetic testing needed (although the increasing availability of whole genome sequencing (genetic screening of the entire genome rather than specific genes in a selected panel) may reduce the need to accurately characterise a condition prior to initiating genetic screening); specialised diagnostic equipment such as electroretinography (ERG) and perimetry are integral to this. ${ }^{9}$ As highlighted in the IRD COI reports for ROI and UK, $73.2 \%$ of persons living with an IRD, and the parents of children (under 18) living with an IRD (75\%), were frustrated by the lack of awareness and support for IRDs. ${ }^{7,8}$ Thus, it is important to create a greater awareness of IRDs in the ophthalmic community (optometrists, orthoptists, ophthalmologists, nurses, allied healthcare professionals) to know what to do for IRD patients - in the first instance reaching out to specialist ophthalmologists (often retinal specialists) who can pick the right tests, interpret the results, answer patient questions, initiate genetic testing, and direct patients and families to further support and information. ${ }^{9}$ Liaising with the ERN-EYE (European Reference Network for Rare Eye Disease) is also to be encouraged and provides a good example of a network of specialists working together on rare eye diseases such as IRDs.

It is well understood that IRDs are complex conditions, but they are also actionable conditions and for patients to take action, so too must those who develop health policy at the national and regional levels. In this era of innovation and possibility, decision makers must understand that access to genetic testing for IRDs is essential in addressing an unmet need. For individuals and families who have been diagnosed with an IRD, a genetic test is a prerequisite in order to make critical life decisions, to 
access the correct care plan, and appropriate clinical trials, as well as emerging therapies.

Patient organisations and clinicians know that access to genetic testing, the delivery of test results, and indeed the type of genetic test patients receive varies enormously. Many patients access to research grade genetic testing and are not aware that there is no obligation on the researcher to provide the results to patients. A number of national genetic testing programmes are funded by charitable organisations and so are unsustainable long term as they rely on donations from the general public. In some countries, testing is available through health insurance, but only if a treatment is available for that condition.

Improvements in the quality and quantity of genetic testing would also benefit the formation of patient registries, natural history studies, assessments of care pathways, and the development of, and enrolment to, clinical trials. ${ }^{18}$ A component required for the success of clinical trials is the adequate measurement of therapeutic impact. A scoping review comprehensively assessing current practice in terms of the outcome measures in clinical trials, highlights that gaps in the assessment of therapy for a variety of ophthalmic diseases exist. ${ }^{33}$ There is increasing acceptance that the patient voice must be heard and that patients are key decision makers in their care, with patients usually placing greater emphasis on the nonclinical aspects of treatment; referred to as the "lived experience". ${ }^{34}$ Patient-reported outcome measures are key parameters that need to be factored in from the beginning. The authors advocate for patient consultation on the design of outcome measures reflective of the lived experience with the disease. Development and use of patientreported outcome measures would support the use of new therapies. Defining success based on clinical and patient factors, such as improvement required for greater quality of life, will make a stronger case for the successful adoption of therapies. ${ }^{34,35}$

Genetic Counselling is an essential to a patient to appropriately interpret genetic test results. A thorough family history can help to better direct genetic testing. Genetic counselling has huge value pre- and post-genetic testing, including in managing expectations particularly in that not every test will find a genetic cause, and that not every genetic cause will have a therapy in the pipeline. ${ }^{9}$

Productivity costs were the largest economic costs associated with IRD, reflecting $£ 8.1$ million in the ROI and $£ 114.1$ million in the UK. In the ROI and the UK, respectively, 74\% ( $£ 5.9$ million), and 78\% ( $£ 89$ million) of this was due to forgone income as a result of reduced workforce participation. ${ }^{7,8}$ The reasons behind this need to be investigated.

Employment levels are lower for those affected with IRDs. In the ROI and the UK, persons with an IRD were $55.7 \%$ and $40.2 \%$, respectively, less likely to be in paid employment than the general population. Lack of employment is a major risk factor for depression and anxiety, making equitable access to employment even more important to be advocated for. Importantly, recent research has shown the significant mental health benefits of just $8 \mathrm{hrs}$ paid employment per week. ${ }^{36}$ Therefore, employment opportunities should be made accessible for those who wish to work, as it may improve levels of negative mental health.

Alarmingly, in the ROI, $24 \%$ of persons living with an IRD and in employment indicated they did not use any additional items to support their work because it was not available to them. ${ }^{7}$ By comparison, in the UK, $5.6 \%$ of persons living with an IRD in employment report not using additional items to support their work because it was not available to them.

Compared to other conditions which have much larger awareness and investment, such as stroke and cancer, IRDs bear a similar cost burden, yet do not receive the same investment. For example, in Ireland, following a survey of patients and carers in 2019 the mean cost of cancer per person per month was estimated at $£ 650$ equating to $£ 7802$ per year, ${ }^{37}$ which works out much lower than the cost per person with an IRD for the same year, $£ 27,988 .^{7}$ Looking at more similar expenditure, in the UK, recent data on the mean health and social care costs for stroke patients per person per year were $£ 22,429,{ }^{38}$ whereas the health and social costs per person with an IRD in 2019 were $£ 25,140 .{ }^{8}$ However, the total cost of health and social care per year for stroke patients in the same study was $£ 3.6$ billion, this was due to the number of stroke cases for the corresponding year $(84,184) .{ }^{38}$ A report by Stroke.org.uk indicated an increase in stroke research spending from $£ 23$ million in $2007 / 2008$ to $£ 55$ million in $2012,{ }^{39}$ this is much less than the $£ 3.1$ million investment in medical research for IRDs in the UK in $2019 .^{8}$

Even though IRDs are life-long debilitating conditions, the measure of their burden both financial and nonfinancial to date has been over-looked and undercalculated. In part, this is due to the lack of fatality associated with these conditions and also due to their 
rarity. Great improvements could be made to the lives of individuals living with IRDs with additional research and investment into causative genes and therapies to circumvent or delay sight loss, and/or medical technologies to enhance living with sight loss.

Different costs of disease are borne by different individuals or sectors of society. Understanding how costs are shared helps to make informed decisions regarding interventions. While persons living with an IRD are most severely affected by the condition, family members and other parts of society also face costs attributable to IRDs. Of the total IRD cost in the ROI for $2019,86 \%$ of this cost is borne by the individuals affected, their families, friends and society. Similarly, in the UK, $66 \%$ of this cost is borne by the individuals affected, their families, friends and society. With regard to society's willingness to pay for reimbursement, it is clear patients and the public already pay the greatest cost of these conditions.

Equally important is that the current methods for therapeutic reimbursement do not adequately incorporate the impact and cost burden to the IRD patient, their families or to society. The burden of disease is borne largely by the individuals affected and the families and friends (Figure 1). This will also need to change to allow the full promise of genetic, pharmacological, technological and regenerative medicine to be realised and transform the lives of millions of patients and families worldwide.

A report from the European Commission indicated wellbeing should take a central place in economic decision-making. ${ }^{27}$ The European Commission concluded a reprioritisation of investment should take into account a more holistic approach to identifying and minimizing decrements in well-being that affect economic growth, including assessment of policies across governmental sectors which contribute to the inequalities in well-being outcomes. $^{27}$ This report also highlights these very points for the IRD community. Approximately one third of costs associated with IRDs were attributable to wellbeing in both regions; in the ROI $32.3 \%$ (£13.8 million), and $37.5 \%$ (£196.1 million) in the UK.

Wellbeing is negatively impacted for those affected with IRDs, their families and their carers. Like other rare diseases, IRDs prove challenging through each stage of the disease and care pathway, this includes challenges obtaining a diagnosis, challenges with day-to-day tasks, and access to care. A report on rare diseases in Europe indicated a large percentage of patients and carers reported difficulty completing daily tasks $(80 \%) .{ }^{28}$ Regarding care,
$60 \%$ of patients and carers found it difficult to manage care, and $70 \%$ found it time-consuming. ${ }^{28}$ It was also reported that health, social, and local services communicated poorly with each other by $67 \%$ of respondents. ${ }^{28}$ In addition to the COI reports for the ROI and UK, IRDs have been shown to impact on physical functioning, quality of life, impacts on daily living including social and emotional functioning, work and education. ${ }^{29-32}$ Research has been conducted in many countries on some of the more prevalent individual IRDs, and on the impacts of individual IRDs. ${ }^{29-32}$ One of the valuable messages from this review is the clear demonstration of the collective impact and burden of IRDs, though individually rare the IRD community faces common issues that must be addressed. This includes the need for timely equitable access to genetic testing and counselling, equality in access to employment, and a revision of the assessment for reimbursement. Actionable improvements in awareness, diagnosis, and access to genetic testing, genetic counselling and access to employment, may all help to minimize the impact of IRDs on well-being.

\section{Conclusion}

In conclusion, the lack of awareness of, and investment in, IRDs have imposed a significant burden to the Irish and UK economies (£42.6 million, £523.3 million). ${ }^{7,8}$ The largest proportion of this being attributable to wellbeing ( $£ 13.8$ million, ROI; £196.1 million, UK), and could be markedly reduced with investment in:

- Research to identify remaining unknown causative genes,

- Research to develop therapies (including genetic, pharmacological, technological, and regenerative medicine),

- Education of all professionals involved in eye care, including optometrists and ophthalmologists, in the identification and management pathways for IRDs,

- Access to genetic testing and genetic counselling,

- Equitable access to education and employment,

- Reassessing and developing policy regarding the methods of reimbursement for the IRD and vision community with regards to treatments and care.

Investment in the above areas would improve the referral pathway and help reduce the frustration of the IRD community $(73.2 \%)$ with the lack of awareness of IRDs, and the negative mental health implications such as anxiety (85.5\%, ROI; 86.8\%, UK) and depression 
(63.2\%, ROI; 67.9\%, UK). ${ }^{7,8}$ In addition, appropriate measures of reimbursement should be developed for the IRD community. Current methods of reimbursement are suitable for conditions for which the population engages with the health system such as stroke and cardiovascular disease with direct health care costs representing $50 \%$, and $60 \%$ of total associated costs, respectively. ${ }^{40,41}$ However, the IRD population do not heavily rely on the health care system with costs reflecting only $4.5 \%$ of costs associated with IRDs in the ROI and $4.8 \%$ of total costs in the UK. ${ }^{7,8}$ It is therefore clear that although individually rare, IRD patients and their families have three major issues in common; the need for timely equitable access to genetic testing and counselling, equality in accessing employment, and, a revision of the assessment process for reimbursement of therapies which currently focuses on the cost-of-illness to the healthcare system. This review demonstrates clearly that IRD patients do not frequently engage the healthcare system and as such suggests that a cost of illness from a societal perspective as outlined in this review may potentially be a better format.

\section{Ethics}

Ethical and patient consent procedures complied with all requirements set out in the European Union General Data Protection Regulations (GDPR), and ethical and compliance obligations.

\section{Data Sharing Statement}

The data described in this article are openly available in the Open Science Framework at DOI:10.17605/OSF.IO/ TPA6U.

\section{Funding}

This study was funded equally by the IRD COUNTS consortium partners who recognized the need to better understand the real impact of inherited retinal dystrophies (IRDs) in the Republic of Ireland (ROI) and, the United Kingdom (UK) of Great Britain and Northern Ireland. IRD COUNTS is a consortium of patient-led organisations, industry and clinician partners who assisted in the design of the project and recruitment of survey participants. IRD COUNTS partners: Fighting Blindness Ireland, Retina UK, Thomas Pocklington Trust, F.Hoffmann-La Roche, MeiraGTx, Novartis Limited UK, ProQR. IRD COUNTS is managed by Retina International.

\section{Disclosure}

Dr Orla Galvin and Ms Avril Daly report grants from Fighting Blindness Ireland, Retina UK, Thomas Pocklington Trust, F. Hoffmann-La Roche, Novartis UK, PROQR, and MeiraGTx, who are equal funders of the multistakeholder consortium which funded this research. Dr Gloria Chi is an employee of Genentech. Dr Laura Brady is an employee of Fighting Blindness Ireland. Ms Marta Del Valle Rubido and Claire Hippert are employees of F. Hoffmann-La Roche Ltd. Prof Michel Michealides Michel consults for MeiraGTx, Acucela, Stargazer Pharma and $2 \mathrm{CTech}$. The authors report no other conflicts of interest in this work.

\section{References}

1. Sergouniotis PI. Inherited retinal disorders: using evidence as a driver for implementation. Ophthalmologica. 2019;5:1-8.

2. Editorial. Spotlight on rare diseases. Lancet Diabetes Endocrinol. 2019;7:2.

3. Richter T, Nestler-Parr S, Babela R, et al. Rare disease terminology and definitions-a systematic global review: report of the ISPOR rare disease special interest group. Value Health. 2015;18(6):906-914. doi:10.1016/j.jval.2015.05.008

4. Daiger SP, Sullivan LS, Bowne SJ. RetNet; 2019. Available from: https:// sph.uth.edu/retnet/sum-dis.htm\#A-genes. Accessed October 3, 2019.

5. Duncan JL, Pierce EA, Laster AM, et al. Inherited retinal degenerations: current landscape and knowledge gaps. Transl Vis Sci Technol. 2018;7(4):6. doi:10.1167/tvst.7.4.6

6. European Medicines Agency. Luxturna; 2019. Available from: https://www.ema.europa.eu/en/medicines/human/EPAR/luxturna. Accessed November 06, 2019.

7. Deloitte Access Economics. The Socioeconomic Impact of Inherited Retinal Dystrophies (Irds) in the Republic of Ireland; 2019.

8. Deloitte Access Economics. The Socioeconomic Impact of Inherited Retinal Dystrophies (Irds) in the United Kingdom; 2019.

9. Stuart A. Inherited retinal disease redefining patient care. Eye Net Mag. 2018;2018:43-48.

10. Davis K, Schoenbaum SC, Audet AMA. 2020 vision of patient-centered primary care. J Gen Intern Med. 2005;20 (10):953-957. doi:10.1111/j.1525-1497.2005.0178.x

11. Mullins CD, Vandigo J, Zheng Z, Wicks P. Patient-centeredness in the design of clinical trials. Value Health. 2014;17(4):471-475. doi:10.1016/j.jval.2014.02.012

12. Moore AT. Genetic testing for inherited retinal disease. Ophthalmology. 2017;124(9):1254-1255. doi:10.1016/j.ophtha.2017.06.018

13. Zhao Y, Feng K, Liu R, Pan J, Zhang L, Lu X. Vitamins and mineral supplements for retinitis pigmentosa. $J$ Ophthalmol. 2019;21 (2019):8524607.

14. Federspiel CA, Bertelsen M, Kessel L. Vitamin A in Stargardt disease-an evidence-based update. Ophthalmic Genet. 2018;39 (5):555-559. doi:10.1080/13816810.2018.1488174

15. Sofi F, Sodi A, Franco F, et al. Dietary profile of patients with Stargardt's disease and retinitis pigmentosa: is there a role for a nutritional approach? BMC Ophthalmol. 2016;16:13-016-0187-3. doi:10.1186/s12886-016-0187-3

16. Maguire AM, Russell S, Wellman JA, et al. Efficacy, safety, and durability of voretigene neparvovec-rzyl in RPE65 mutation-associated inherited retinal dystrophy: results of Phase 1 and 3 trials. Ophthalmology. 2019;126(9):1273-1285. doi:10.1016/j. ophtha.2019.06.017 
17. Whelan L, Dockery A, Wynne N, Kenna PF, Farrar GJ. Findings from a genotyping study of over 1000 people with inherited retinal disorders in Ireland. Genes. 2020;11:105. doi:10.3390/genes11010105

18. Gliklich R, Dreyer N, Leavy M, editors. Registries for Evaluating Patient Outcomes: A User's Guide. Patient Registries. 3rd ed. Rockville (MD): Agency for Healthcare Research and Quality (US); Apr 1, 2014. Available from: https://www.ncbi.nlm.nih.gov/ books/NBK208643/. Accessed February 19, 2020.

19. Zhang Y, Wang Z, Huang S, et al. Parents' perceptions of diagnostic genetic testing for children with inherited retinal disease in China Mol Genet Genomic Med. 2019;7(9):e916. doi:10.1002/mgg3.v7.9

20. Weil J. Genetic counselling in the era of genomic medicine. As we move towards personalized medicine, it becomes more important to help patients understand genetic tests and make complex decisions about their health. EMBO Rep. 2002;3(7):590-593. doi:10.1093/ embo-reports/kvf144

21. Garcia GA, Khoshnevis M, Gale J, et al. Profound vision loss impairs psychological well-being in young and middle-aged individuals. Clin Ophthalmol. 2017;22(11):417-427. doi:10.2147/OPTH.S113414

22. Cumberland PM, Rahi JS; UK Biobank Eye and Vision Consortium. Visual function, social position, and health and life chances: the UK biobank study. JAMA Ophthalmol. 2016;134(9):959-966. doi:10.1001/jamaophthalmol.2016.1778

23. van den Heuvel SG, Geuskens GA, Hooftman WE, Koppes LL, van den Bossche SN. Productivity loss at work; health-related and work-related factors. J Occup Rehabil. 2010;20(3):331-339. doi:10.1007/s10926-009-9219-7

24. Baxter K, Rabiee P. Estimating the Unit Costs of Vision Rehabilitation Services. Unit Costs of Health and Social Care 2015. PSSRU University of Kent; 2015:16-21.

25. Health Service Executive (HSE). Health Sector: Consolidated Salary Scales in Accordance with FEMPI 2015 and the Public Service Stability Agreements 2013-2020 (The Lansdowne Road Agreements); 2019. Available from: https://www.hse.ie/eng/staff/ben efitsservices/pay/. Accessed February 19, 2020.

26. OECD. Health at a Glance 2017: OECD Indicators; 2017. doi:10.1787/health_glance-2017-en

27. General Secretariat of the Council of the European Union. The economy of well-being: executive summary of the OECD background paper on "creating opportunities for people's wellbeing and economic growth"; June 21st, 2019. Available from: https://data.consilium.europa.eu/doc/ document/ST-10414-2019-INIT/en/pdf. Accessed December 4th, 2019.

28. Courbier S, Berjonneau E. Juggling Care and Daily Life: The Balancing Act of the Rare Disease Community. May ed. Paris: EURORDIS-Rare Diseases Europe; 2017.
29. Chaumet-Riffaud AE, Chaumet-Riffaud P, Cariou A, et al. Impact of retinitis pigmentosa on quality of life, mental health, and employment among young adults. Am J Ophthalmol. 2017;177:169-174. doi:10.1016/j.ajo.2017.02.016

30. Garip G, Kamal A. Systematic review and meta-synthesis of coping with retinitis pigmentosa: implications for improving quality of life. BMC Ophthalmol. 2019;19(1):181. doi:10.1186/s12886-0191169-z

31. Pennesi ME, Birch DG, Duncan JL, Bennett J, Girach A. CHOROIDEREMIA: retinal degeneration with an unmet need. Retina. 2019;39:2059-2069.

32. Tolley C, Mullins A, Kilgariff S, et al. Qualitative interviews to inform development of a Patient Reported Outcome (PRO) strategy in RLBP1 retinitis pigmentosa (RLBP1 RP). Value Health. 2017;20 (9):A761. doi:10.1016/j.jval.2017.08.2157

33. Jolly J, Bridge H, MacLaren R. Outcome measures used in ocular gene therapy trials: a scoping review of current practice. Front Pharmacol. 2019;10:1076.

34. Dean S, Mathers JM, Calvert M, et al. "The patient is speaking": discovering the patient voice in ophthalmology. $\mathrm{Br} J$ Ophthalmol. 2017;101(6):700-708. doi:10.1136/bjophthalmol-2016-309955

35. McGlothlin AE, Lewis RJ. Minimal clinically important difference: defining what really matters to patients. JAMA. 2014;312 (13):1342-1343. doi:10.1001/jama.2014.13128

36. Kamerade D, Wang S, Burchell B, Balderson SU, Coutts A. A shorter working week for everyone: how much paid work is needed for mental health and well-being? Soc Sci Med. 2019;241:112353. doi:10.1016/j.socscimed.2019.06.006

37. The Irish Cancer Society. Irish Cancer Society-Real Cost of Cancer 2019 Report; 2019.

38. Xu XM, Vestesson E, Paley L, et al. The economic burden of stroke care in England, Wales and Northern Ireland: using a national stroke register to estimate and report patient-level health economic outcomes in stroke. Eur Stroke J. 2018;Mar(1):82-91. doi:10.1177/ 2396987317746516

39. Luengo-Fernandez R, Leal JGA. Research spend in the UK Comparing stroke, cancer, coronary heart disease and dementia. Rebuilding Lives After Stroke. doi:10.1136/bmjopen-2014-006648

40. Saka O, McGuire A, Wolfe C. Cost of stroke in the United Kingdom. Age Ageing. 2009;38(1):27-32. doi:10.1093/ageing/ afn281

41. Luengo-Fernandez R, Leal J, Gray A, Petersen S, Rayner M. Cost of cardiovascular diseases in the United Kingdom. Heart. 2006;92 (10):1384-1389. doi:10.1136/hrt.2005.072173
Clinical Ophthalmology

\section{Publish your work in this journal}

Clinical Ophthalmology is an international, peer-reviewed journal covering all subspecialties within ophthalmology. Key topics include: Optometry; Visual science; Pharmacology and drug therapy in eye diseases; Basic Sciences; Primary and Secondary eye care; Patient Safety and Quality of Care Improvements. This journal is indexed on PubMed
Central and CAS, and is the official journal of The Society of Clinical Ophthalmology (SCO). The manuscript management system is completely online and includes a very quick and fair peer-review system, which is all easy to use. Visit http://www.dovepress.com/ testimonials.php to read real quotes from published authors. 